\title{
Control of Systems with Arbitrary Bounded Input Delay Using Implicit Lyapunov Function Technique*
}

\author{
Konstantin Zimenko ${ }^{1}$, Denis Efimov ${ }^{1,2}$, Andrey Polyakov ${ }^{1,2}$ and Artem Kremlev ${ }^{1}$
}

\begin{abstract}
The paper presents control algorithms for systems with input delay. There are two main results based on using Implicit Lyapunov Function (ILF) technique: 1) an LMI-based approach is presented to evaluate the domain of attraction of a finite-time stable control in the case of the arbitrary bounded delayed control input; 2) a uniting control is designed with commutation between two laws providing a global boundedness of all trajectories of systems with any input delay, and convergence to the origin for a sufficiently small one. The results are also preserved for the time-varying delay case. The theoretical results are supported by numerical examples.
\end{abstract}

\section{INTRODUCTION}

Control development for plants with delay has received increasing attention in the very recent years, due to the wide use of teleoperated and networked systems (see e.g. [1]-[7] to name a few). The lags and delays usually appear in robotic remote control systems, aerospace and seabed applications, medicine (for instance, tele-surgery), etc. For example, for implementation of bilateral haptic teleoperation it is natural, that the transmission of commands and sensor signals is affected with the time delay in the range $\sim\left(10^{-3}-10^{-1}\right)$ seconds for terrestrial telerobotics applications and up to several seconds for inner space applications [6].

Such an attention is due the time-delays can be a source of system instability in many cases. Therefore, stability analysis and development of control algorithms, which are robust with respect to uncertain and time-varying delays, are of theoretical and practical importance. Despite of variety of methods solving described problem, most of them deal with linear control systems. Constructive and computationally tractable conditions exist for linear time-delay models only [1].

Another motivating point for studying this topic is that modern control algorithms are mostly digitally implemented, i.e. in applications sampled-data control naturally appears. According to [8], [4] a sampled-data control system can be represented as a system with time-varying delay in control channel, where the delay is piecewise linear (sawtooth).

*This work is supported by the Russian Science Foundation under grant 17-19-01422.

${ }^{1}$ Konstantin Zimenko, Andrey Polyakov, Denis Efimov and Artem Kremlev are with Department of Control Systems and Informatics, ITMO University, 49 Kronverkskiy av., 197101 Saint Petersburg, Russia.

${ }^{2}$ Andrey Polyakov and Denis Efimov are with Non-A INRIA - LNE, Parc Scientifique de la Haute Borne 40, avenue Halley Bat.A, Park Plaza 59650 Villeneuve d'Ascq They are also with CRIStAL (UMR-CNRS 9189), Ecole Centrale de Lille, BP 48, Cite Scientifique, 59651 Villeneuve-d'Ascq, France.

e-mail: kostyazimenko@gmail.com, denis.efimoveinria.fr, andrey.polyakoveinria.fr, kremlev_artem@mail.ru
The present paper addresses robustness analysis of Implicit Lyapunov Function (ILF)-based control system introduced in [23]. It has been shown, that ILF control algorithm preserves boundedness of the system trajectories for any delay and guarantees finite-time stability of the origin in absence of delay. Based on this result there were developed ILF-based control algorithms, which preserve asymptotic stability for small delays $0<\tau<\tau_{0}$ and global asymptotic stability with respect to a compact set containing the origin for $\tau \geq \tau_{0}$. Presented algorithms can be used for the case of the fastvarying delay $\tau(t)$. Such control algorithms may be useful, for example, when the data transmission channel is unreliable and fickle (for instance, the delay of data transmission depends on the number of network users, there are packet losses, etc.). In the given work that scheme is augmented by a supervision algorithm, which activates a linear feedback guaranteeing stability for delays in prescribed limits, and the nonlinear ILF control from [23] is switched on if a possible instability is detected.

Notation: The symbol $\overline{1, m}$ is used to denote a sequence of integers $1, \ldots, m . \mathbb{R}^{n}$ denotes the $n$ dimensional Euclidean space with vector norm $|\cdot|, \mathbb{R}_{>}=\{s \in \mathbb{R}: s>0\}, \mathbb{R}_{\geq}=$ $\{s \in \mathbb{R}: s \geq 0\}, \operatorname{diag}\left\{\lambda_{i}\right\}_{i=1}^{n}$ is the diagonal matrix with the elements $\lambda_{i}$ on the main diagonal, the notation $P>0$, for $P \in \mathbb{R}^{n \times n}$ means that $P$ is symmetric and positive definite, $p_{i j}, i, j=\overline{1, n}$ are the entries in the $i$-th row and $j$-th column of $P$, the minimal and maximal eigenvalues of $P$ are denoted by $\lambda_{\min }(P)$ and $\lambda_{\max }(P)$, respectively. The Banach space of continuous functions $\phi:[-\tau, 0] \rightarrow \mathbb{R}^{n}$ with the uniform norm $\|\phi\|=\sup _{-\tau \leq \varsigma \leq 0}|\phi(\varsigma)|$ is denoted by $C_{[-\tau, 0]}$.

\section{PROBLEM FORMULATION}

Consider a single input control system of the form

$$
\dot{x}(t)=A x(t)+b u(t)+d(t, x(t)),
$$

where $x(t) \in \mathbb{R}^{n}$ is the state vector, $u(t) \in \mathbb{R}$ is the control input,

$$
A=\left(\begin{array}{ccccc}
0 & 1 & 0 & \cdots & 0 \\
0 & 0 & 1 & \cdots & 0 \\
\vdots & \vdots & \vdots & \ddots & \vdots \\
0 & 0 & 0 & \cdots & 1 \\
0 & 0 & 0 & \cdots & 0
\end{array}\right) \quad \text { and } \quad b=\left(\begin{array}{c}
0 \\
0 \\
\vdots \\
0 \\
1
\end{array}\right)
$$

$d: \mathbb{R}^{n+1} \rightarrow \mathbb{R}^{n}$ describes the system nonlinearities, uncertainties and disturbances, which may be non-Lipschitz or discontinuous. Note, that presented system appears for a number of control applications since many controllable single-input nonlinear systems can be transformed to a canonical form like (1) (see, for example, [9]). 
Assume that there is a delay $\tau$ in the control (and/or measurement) channel, i.e.

$$
\dot{x}=A x(t)+b u(t-\tau)+d(t, x),
$$

or retreating $\int_{t-\tau}^{t} \dot{u}(s) d s$ as a disturbance

$$
\dot{x}=A x(t)+b u(t)+d(t, x)+d_{\tau}(t),
$$

where $d_{\tau}(t)=-b \int_{t-\tau}^{t} \dot{u}(s) d s$.

The aim of the paper is to develop the stabilizing control algorithms for the system (2), which

- provide finite-time attractiveness of some compact set around the origin for any delay $\tau$ (for $\tau=0$ the origin is finite-time stable);

- provide asymptotic stability of the system for the delay $\tau<\tau_{0}, \tau_{0} \in \mathbb{R}_{>}$and preserve boundedness of the system trajectories for any delay $\tau \geq \tau_{0}$.

\section{PRELIMINARIES}

Let us consider the system

$$
\dot{x}=f(x), \quad x(0)=x_{0},
$$

where $x \in \mathbb{R}^{n}$ is the state vector, $f \in \mathbb{R}^{n} \rightarrow \mathbb{R}^{n}$ is a vector field, $f(0)=0$. If the vector field $f$ is discontinuous with respect to $x$, then the solutions $x\left(t, x_{0}\right)$ of (3) fall into the area of differential inclusions and need to be understood in the sense of Filippov [10].

\section{A. Finite-Time Stability}

Definition 1 [24], [15] The origin of (3) is said to be globally finite-time stable if it is globally asymptotically stable and any solution $x\left(t, x_{0}\right)$ of (3) reaches the equilibrium point at some finite time moment, i.e. $x\left(t, x_{0}\right)=0 \forall t \geq$ $T\left(x_{0}\right)$, where $T: \mathbb{R}^{n} \rightarrow \mathbb{R}_{\geq}$is the settling-time function.

Theorem 1 [24], [25] Suppose there exists a positive definite $C^{1}$ function $V$ defined on an open neighborhood of the origin $D \subset \mathbb{R}^{n}$ and real numbers $C>0$ and $\sigma \geq 0$, such that the following condition is true for the system (3)

$$
\dot{V}(x) \leq-C V^{\sigma}(x), \quad x \in D \backslash\{0\} .
$$

Then depending on the value $\sigma$ the origin is stable with different types of convergence:

- if $\sigma=1$, the origin is asymptotically stable;

- if $0 \leq \sigma<1$, the origin is finite-time stable and

$$
T\left(x_{0}\right) \leq \frac{1}{C(1-\sigma)} V_{0}^{1-\sigma},
$$

where $V_{0}=V\left(x_{0}\right)$;

- if $\sigma>1$ the origin is asymptotically stable and, for every $\epsilon$, the set $B(0, \epsilon)=\{x \in D: V(x)<\varepsilon\}$ is fixedtime (independent on the initial values) attractive with

$$
T\left(x_{0}\right) \leq T_{\max }=\frac{1}{C(\sigma-1) \varepsilon^{\sigma-1}} .
$$

If $D=\mathbb{R}^{n}$ and function $V$ is radially unbounded, then the system (3) admits these properties globally.

\section{B. Implicit Lyapunov Function Method}

The following theorem presents the ILF method [16], [17] for finite-time stability analysis.

Theorem 2 [23] If there exists a continuous function

$$
\begin{aligned}
& Q: \mathbb{R}_{>} \times \mathbb{R}^{n} \rightarrow \mathbb{R} \\
& (V, x) \mapsto Q(V, x)
\end{aligned}
$$

such that

C1) $Q(V, x)$ is continuously differentiable $\forall x \in \mathbb{R}^{n} \backslash\{0\}$ and $\forall V \in \mathbb{R}_{>}$;

C2) for any $x \in \mathbb{R}^{n} \backslash\{0\}$ there exist $V^{-} \in \mathbb{R}_{>}$and $V^{+} \in \mathbb{R}_{>}$.

$$
Q\left(V^{-}, x\right)<0<Q\left(V^{+}, x\right)
$$

C3) for $\Omega=\left\{(V, x) \in \mathbb{R}^{n+1}: Q(V, x)=0\right\}$

$$
\lim _{\substack{x \rightarrow 0 \\(V, x) \in \Omega}} V=0, \lim _{\substack{V \rightarrow 0^{+} \\(V, x) \in \Omega}}\|x\|=0, \lim _{\substack{\|x\| \rightarrow \infty \\(V, x) \in \Omega}} V=+\infty ;
$$

C4) the inequality $-\infty<\frac{\partial Q(V, x)}{\partial V}<0$ holds $\forall V \in \mathbb{R}_{>}$and $\forall x \in \mathbb{R}^{n} \backslash\{0\}$;

C5) the inequality

$$
\frac{\partial Q(V, x)}{\partial x} f(x) \leq h V^{1-\mu} \frac{\partial Q(V, x)}{\partial V}
$$

holds $\forall(V, x) \in \Omega$, where $0<\mu \leq 1$ and $h>0$ are some constants.

Then the origin of the system (3) is globally finite-time stable with the following settling time estimate

$$
T\left(x_{0}\right) \leq \frac{V_{0}^{\mu}}{h \mu}
$$

Based on this result in [23] a finite-time stabilizing control law has been developed for the system (1).

Introduce the implicitly defined Lyapunov function by

$$
Q(V, x)=x^{T} D\left(V^{-1}\right) P D\left(V^{-1}\right) x-1,
$$

where $D(\lambda)$ is the diagonal matrix of the form $D(\lambda)=$ $\operatorname{diag}\left\{\lambda^{1+(n-i) \mu}\right\}_{i=1}^{n}$ and $P \in \mathbb{R}^{n \times n}$ is a symmetric positive definite matrix. Denote the diagonal matrix $H_{\mu}=\operatorname{diag}\{1+$ $(n-i) \mu\}_{i=1}^{n}$.

Theorem 3 [23], [14] If

- the system of matrix inequalities

$$
\left\{\begin{array}{l}
A X+X A^{T}+b y+y^{T} b^{T}+L+R \leq 0, \\
\frac{1}{\alpha} L \geq X H_{\mu}+H_{\mu} X>0, \quad X>0,
\end{array}\right.
$$

is feasible for some $R \in \mathbb{R}^{n \times n}, R>0, \mu \in(0,1]$, $\alpha \in \mathbb{R}_{>}, X \in \mathbb{R}^{n \times n}$ and $y \in \mathbb{R}^{1 \times n}$;

- the control has the form

$$
u(V, x)=V^{1-\mu} k D\left(V^{-1}\right) x,
$$

where $k=y X^{-1}$,

$$
V \in \mathbb{R}_{>}: Q(V, x)=0
$$

and $Q(V, x)$ is defined by (5) with $P=X^{-1}$;

- the disturbance function $d(t, x)$ satisfy

$$
\begin{array}{r}
d^{T}(t, x) D\left(V^{-1}\right) R^{-1} D\left(V^{-1}\right) d(t, x) \leq \\
\beta V^{-2 \mu} x^{T} D\left(V^{-1}\right) P L P D\left(V^{-1}\right) x
\end{array}
$$


with $\beta \in(0,1)$ and $(t, x) \in \mathbb{R}_{>} \times \mathbb{R}^{n}$.

Then the closed-loop system (1), (7) is globally finite-time stable and the settling time function estimate has the form

$$
T\left(x_{0}\right) \leq \frac{V_{0}^{\mu}}{\alpha \mu(1-\beta)},
$$

where $V_{0} \in \mathbb{R}_{>}: Q\left(V_{0}, x_{0}\right)=0$.

\section{Delay Robustness for Homogeneous Systems}

Consider an autonomous functional differential equation of retarded type [21]:

$$
\dot{x}(t)=f\left(x_{t}\right), t \geq 0,
$$

where $x(t) \in \mathbb{R}^{n}$ and $x_{t} \in C_{[-\tau, 0]}$ is the state function, $x_{t}(s)=x(t+s)$ for $s \in[-\tau, 0], f: C_{[-\tau, 0]} \rightarrow \mathbb{R}^{n}$ is continuous and ensures existence and uniqueness of solutions in forward time [21], $f(0)=0$. We assume that the system (10) for the initial functional condition $x_{0} \in C_{[-\tau, 0]}$ has a unique solution.

For any $r_{i}>0, i=\overline{1, n}$ and $\lambda>0$, define the dilation matrix $\Lambda_{r}(\lambda)=\operatorname{diag}\left\{\lambda^{r_{i}}\right\}_{i=1}^{n}$ and the vector of weights $r=\left[r_{1}, \ldots, r_{n}\right]^{T}$.

For any $r_{i}>0, i=\overline{1, n}$ and $x \in \mathbb{R}^{n}$ the homogeneous norm can be defined as follows [19], [11]

$$
|x|_{r}=\left(\sum_{i=1}^{n}\left|x_{i}\right|^{\rho / r_{i}}\right)^{1 / \rho}, \rho \geq \max _{1 \leq i \leq n} r_{i} .
$$

The homogeneous norm has an important property that is $\left|\Lambda_{r}(\lambda) x\right|_{r}=\lambda|x|_{r}$ for all $x \in \mathbb{R}^{n}$.

For any $r_{i}>0, i=\overline{1, n}$ and $\phi \in C_{[-\tau, 0]}$ the homogeneous norm can be defined as follows

$$
\|\phi\|_{r}=\left(\sum_{i=1}^{n}\left\|\phi_{i}\right\|^{\rho / r_{i}}\right)^{1 / \rho}, \rho \geq \max _{1 \leq i \leq n} r_{i} .
$$

The homogeneous norm in the Banach space has the same important property that $\left\|\Lambda_{r}(\lambda) \phi\right\|_{r}=\lambda\|\phi\|_{r}$ for all $\phi \in$ $C_{[-\tau, 0]}$. Define $B_{\rho}^{\tau}=\left\{\phi \in C_{[-\tau, 0]}:\|\phi\|_{r} \leq \rho\right\}$ as a closed ball of radius $\rho>0$ in $C_{[-\tau, 0]}$.

Definition 2 [18] The function $g: C_{[-\tau, 0]} \rightarrow \mathbb{R}$ (vector field $f: C_{[-\tau, 0]} \rightarrow \mathbb{R}^{n}$ ) is called $r$-homogeneous with $r_{i}>$ $0, i=\overline{1, n}$, if for any $\phi \in C_{[-\tau, 0]}$ the relation $g\left(\Lambda_{r}(\lambda) \phi\right)=$ $\lambda^{d} g(\phi)\left(f\left(\Lambda_{r}(\lambda) \phi\right)=\lambda^{d} \Lambda_{r}(\lambda) f(\phi)\right)$ holds for some $d \in \mathbb{R}$ $\left(d \geq-\min _{1 \leq i \leq n} r_{i}\right)$ and all $\lambda>0$.

In both cases, the constant $d$ is called the degree of homogeneity. The system (10) is called $r$-homogeneous of degree $d$ if the function $f$ admits this property.

The introduced notion of weighted homogeneity in $C_{[-\tau, 0]}$ is reduced to the standard one in $\mathbb{R}^{n}$ if $\tau=0$.

Theorem 4 [19] Let $f: \mathbb{R}^{n} \rightarrow \mathbb{R}^{n}$ be defined on $\mathbb{R}^{n}$ and be a continuous $r$-homogeneous vector field with degree $d(d<0)$. If the origin of the system (3) is locally asymptotically stable then it is globally asymptotically stable (globally finite-time stable) and there exists a continuously differentiable Lyapunov function $V$ which is $r$-homogeneous of degree $v>-d$.
By definition of homogeneity there exist constants $c_{1}, c_{2} \in$ $\mathbb{R}_{>}$such that

$$
c_{1}\|x\|_{r}^{v} \leq V(x) \leq c_{2}\|x\|_{r}^{v}
$$

For example, the system (1), (7) for $d(t, x)=0$ is $r$ homogeneous of degree $-\mu$ with $r=(1+(n-1) \mu, 1+(n-$ $2) \mu, \ldots, 1)$ and the implicitly defined Lyapunov function is homogeneous of degree 1 , where the parameters $c_{1}$ and $c_{2}$ can be estimated as follows

$$
\begin{aligned}
& c_{1}=\min \left\{\frac{\lambda_{\min }^{\frac{1}{2 r_{\max }}}(P)}{n^{\frac{1}{\rho}}}, \frac{\lambda_{\min }^{\frac{1}{2 r_{\min }}}(P)}{n^{\frac{1}{\rho}}}\right\}, \\
& c_{2}=\max \left\{\left(n \lambda_{\max }(P)\right)^{\frac{1}{2 r_{\max }}},\left(n \lambda_{\max }(P)\right)^{\frac{1}{2 r_{\min }}}\right\} .
\end{aligned}
$$

In [20], [22], [12], [13] robustness with respect to delays has been discussed for homogeneous systems.

Lemma 1 [20] Let $f\left(x_{\tau}\right)=F[x(t), x(t-\tau)]$ in (10) and the system (10) be r-homogeneous with degree $d \geq 0$ and globally asymptotically stable at the origin for $\tau=0$, then for any $\rho>0$ there is $0<\tau_{0}<+\infty$ such that (10) is asymptotically stable at the origin in $B_{\rho}^{\tau}$ for any delay $0 \leq \tau \leq \tau_{0}$.

Lemma 2 [22] Let $f\left(x_{t}\right)=F[x(t), x(t-\tau)]$ in (10) be uniformly continuous and the system (10) be r-homogeneous with degree $d<0$ and globally asymptotically stable at the origin for $\tau=0$, then for any $\varepsilon>0$ there is $0<\tau_{0}<+\infty$ such that (10) is globally asymptotically stable with respect to $B_{\varepsilon}^{\tau}$ for any delay $0 \leq \tau \leq \tau_{0}$.

Thus, (10) is locally robustly stable with respect to a sufficiently small delay if it is $r$-homogeneous with a nonnegative degree and stable in the delay-free case.

\section{MAIN RESULTS}

In this section, first, we provide an LMI-based approach to evaluate the domain of attraction of a finite-time stable control (7) in the case of the delayed plant in (2). Second, a uniting control is designed with commutation between two laws providing a global bounded of all trajectories in (2) for any delay, and convergence to the origin for a sufficiently small one.

A. Control Providing Boundedness of The System Trajectories in Presence of Delay

Lemma 3 (Disturbance-free case) Let the system of linear matrix inequalities

$$
\left\{\begin{array}{l}
A X+X A^{T}+b y+y^{T} b^{T}+L+R \leq 0 \\
\iota X \geq A X+X A^{T} \geq-\iota X \\
X H_{\mu}+H_{\mu} X \geq \eta X, \quad X>0 \\
\left(\begin{array}{cc}
X & y^{T} \\
y & \zeta
\end{array}\right) \geq 0, \quad L \geq \varpi X
\end{array}\right.
$$

be feasible for $R=\left(\begin{array}{cc}0_{n-1} & 0 \\ 0 & r_{n n}\end{array}\right)$ and some $\mu \in(0,1]$, $r_{n n}, \eta, \iota, \zeta, \varpi \in \mathbb{R}_{>}, X \in \mathbb{R}^{n \times n}, y \in \mathbb{R}^{1 \times n}$. The system (2), (7) with $d(t, x)=0$ is globally asymptotically stable with respect to $B_{c / c_{1}}^{2 \tau}$ for any $c \in \mathbb{R}_{>}$if

$$
\tau<\frac{\sqrt{r_{n n} \varpi} c^{\mu}}{\eta^{-1} \sqrt{l_{1}}\left(\iota+2 \sqrt{p_{n n} \zeta}\right)+\sqrt{l_{2}}+\left|k_{n}\right| \sqrt{\zeta}},
$$


where $P=X^{-1}, \quad k=y X^{-1}, \quad l_{1}=$ $\lambda_{\max }\left(X^{1 / 2}\left((1-\mu) I+H_{\mu}\right) k^{T} k\left((1-\mu) I+H_{\mu}\right) X^{1 / 2}\right)$, $l_{2}=\lambda_{\max }\left(X^{1 / 2} A^{T} k^{T} k A X^{1 / 2}\right)$.

Note that in absence of the delay $(\tau=0)$ the origin is finite-time stable.

Remark 1 From the results presented in Lemma 3, knowing the value of delay, one can find the compact set $B_{c / c_{1}}^{2 \tau}$ the system asymptotically converging to. And vise versa, for a given $c$ one can easily find a value $\tau_{0}$ such that for any $\tau<\tau_{0}$ the system (2), (7) is globally asymptotically stable with respect to $B_{c / c_{1}}^{2 \tau}$.

Remark 2 The result of Lemma 3 holds for the case of time-varying delay and sampled-data control realization.

Remarks 1, 2 remain true for the all subsequent results presented below.

Corollary 1 The system (2), (7) can be asymptotically stabilized with respect to an arbitrary small set for any arbitrary bounded delay by appropriate choosing the parameter $c$ and changing the matrix variable $\tilde{P}=P / v, v \in \mathbb{R}_{>}$.

Example 1 Consider the system (2) for $n=3$ in the disturbance-free case $(d(t, x(t))=0)$. Define ILF-based control in the form (7) with the parameter $\mu=0.5$. The matrix $P>0$ and the vector $k$ are obtained from the system (13) with $r_{n n}=0.99, \eta=1.2$ :

$$
\begin{gathered}
P=\left(\begin{array}{lll}
1.0169 & 2.0324 & 1.0147 \\
2.0324 & 5.0786 & 3.0433 \\
1.0147 & 3.0433 & 3.0427
\end{array}\right), \\
k=\left(\begin{array}{lll}
-1.0035 & -3.0089 & -3.0065
\end{array}\right) .
\end{gathered}
$$

Obtained value of maximal delay for $c=1$ is $\tau_{0}=3.75 \times$ $10^{-2}$. The estimation $c_{1}=0.2076$ is obtained in accordance with (12). Then the system (2), (7) is globally asymptotically stable with respect to $B_{4.8174}^{2 \tau}$ for any delay $\tau<\tau_{0}=3.75 \times 10^{-2}$.

It is easy to extend Lemma 3 for disturbed case.

Lemma 4 (Disturbed case) Let the system of linear matrix inequalities

$$
\left\{\begin{array}{l}
\kappa\left(A X+X A^{T}+b y+y^{T} b^{T}\right)+L_{1}+R_{1} \leq 0 \\
(1-\kappa)\left(A X+X A^{T}+b y+y^{T} b^{T}\right)+L_{2}+R_{2} \leq 0 \\
\iota X \geq A X+X A^{T} \geq-\iota X, \quad\left(\begin{array}{cc}
X & y^{T} \\
y & \zeta
\end{array}\right) \geq 0 \\
X H_{\mu}+H_{\mu} X \geq \eta X, \quad X>0, \\
L_{1} \geq \varpi_{1} X, \quad L_{2} \leq \varpi_{2} X, \quad R_{2} \leq \varpi_{3} X,
\end{array}\right.
$$

be feasible for $R_{1}=\left(\begin{array}{cc}0_{n-1} & 0 \\ 0 & r_{n n}\end{array}\right)$ and some $\mu \in(0,1]$, $\kappa \in(0,1), r_{n n}, \eta, \zeta, \iota, \varpi_{1}, \varpi_{2}, \varpi_{3} \in \mathbb{R}_{>}, R_{2}>0, L_{1}>0$, $L_{2}>0, X \in \mathbb{R}^{n \times n}, y \in \mathbb{R}^{1 \times n}$ and the inequality

$$
\begin{aligned}
& d^{T}(t, x(t)) D\left(V^{-1}(t)\right) R_{2}^{-1} D\left(V^{-1}(t)\right) d(t, x(t)) \leq \\
& \beta V^{-2 \mu}(t) x^{T}(t) D\left(V^{-1}(t)\right) P L_{2} P D\left(V^{-1}(t)\right) x(t)
\end{aligned}
$$

holds for $\beta \in(0,1)$ with $V(t)>c$. The system (2), (7) is globally asymptotically stable with respect to $B_{c / c_{1}}^{2 \tau}$ for any $c \in \mathbb{R}_{>}$if

$$
\tau<\frac{\sqrt{r_{n n} \varpi_{1}} c^{\mu}}{\eta^{-1} \sqrt{l_{1}}\left(\iota+2 \sqrt{p_{n n} \zeta}+2 \sqrt{\varpi_{2} \varpi_{3}}\right)+\sqrt{l_{2}}+\left|k_{n}\right| \sqrt{\zeta}+\sqrt{\zeta \varpi_{2} \varpi_{3}}},
$$

where $P=X^{-1}, \quad k=y X^{-1}, \quad l_{1}=$ $\lambda_{\max }\left(X^{1 / 2}\left((1-\mu) I+H_{\mu}\right) k^{T} k\left((1-\mu) I+H_{\mu}\right) X^{1 / 2}\right)$, $l_{2}=\lambda_{\max }\left(X^{1 / 2} A^{T} k^{T} k A X^{1 / 2}\right)$.

Note that if $\tau=0$ and the inequality (16) holds globally, then the origin is finite-time stable.

Remark 3 If the matching condition $d_{i}(t, x(t))=0, i=$ $1, \ldots, n-1$ holds, then to relax the system of LMIs (15) the matrix $R_{2}$ can be chosen as $R_{2}=\left(\begin{array}{cc}0_{n-1} & 0 \\ 0 & \bar{r}_{n n}\end{array}\right), \bar{r}_{n n} \in \mathbb{R}_{>}$.

Remark 4 Considering the case of sampled-data control realization for the system (1) by Lemma 3 (or Lemma 4) global attractiveness of a compact set containing the origin can be shown only. In [23] this issue was studied more precisely where global asymptotic stability of the origin is proven.

Example 2 Consider the system (2) for $n=3$ in the case of matched disturbances $d_{1}(t, x(t))=0, d_{2}(t, x(t))=0$, $d_{3}(t, x(t))=0.2$. Define the control (7) with the parameter $\mu=1$. The parameters of the control (7) were selected solving the LMIs (15) for $r_{n n}=0.7911, \bar{r}_{n n}=0.1990$, $\kappa=0.799, \eta=1$ :

$$
\begin{gathered}
P=\left(\begin{array}{ccc}
1.0072 & 2.0159 & 1.0087 \\
2.0159 & 5.0414 & 3.0274 \\
1.0087 & 3.0274 & 3.026
\end{array}\right), \\
k=\left(\begin{array}{lll}
-0.9988 & -2.9976 & -2.9987
\end{array}\right) .
\end{gathered}
$$

It can be easily checked that the inequality (16) holds. The estimation $c_{1}=0.2475$ is obtained in accordance with (12). Then, according to Lemma 4 the system (2), (7) is globally asymptotically stable with respect to $B_{c / c_{1}}^{2 \tau}=B_{20.1985}^{2 \tau}(c=$ 5) for any delay $\tau \leq \tau_{0}=0.131$.

\section{B. Control Providing Asymptotic Stability in Presence of Delay}

Using of Lemma 3 and Lemma 4 allows to design new hybrid stabilization algorithms, which preserve asymptotic stability of system trajectories.

Consider two implicit Lyapunov functions defined by

$$
\begin{aligned}
& Q_{1}(V, x)=x^{T} D_{\mu}\left(V^{-1}\right) P D_{\mu}\left(V^{-1}\right) x-1, \\
& Q_{2}(V, x)=x^{T} D_{\nu}\left(V^{-1}\right) P D_{\nu}\left(V^{-1}\right) x-1,
\end{aligned}
$$

where $P \in \mathbb{R}^{n \times n}, P>0, D_{\mu}(\lambda)=\operatorname{diag}\left\{\lambda^{1+(n-1) \mu}\right\}_{i=1}^{n}$ and $D_{\nu}(\lambda)=\operatorname{diag}\left\{\lambda^{1+(i-1) \nu}\right\}_{i=1}^{n}$. Denote $H_{\mu}=\operatorname{diag}\{1+$ $(n-i) \mu\}_{i=1}^{n}$ and $H_{\nu}=\operatorname{diag}\{1+(i-1) \nu\}_{i=1}^{n}$.

Then the following results can be obtained.

Theorem 5 (Disturbance-free case) Let for (2), $d(t, x(t))=0$ the system of linear matrix inequalities

$$
\left\{\begin{array}{l}
A X+X A^{T}+b y+y^{T} b^{T}+L_{\mu}+R_{\mu} \leq 0, \\
A X+X A^{T}+b y+y^{T} b^{T}+L_{\nu}+R_{\nu} \leq 0, \\
\iota X \geq A X+X A^{T} \geq-\iota X, \quad\left(\begin{array}{cc}
X & y^{T} \\
y & \zeta
\end{array}\right) \geq 0 \\
X H_{\mu}+H_{\mu} X \geq \eta_{\mu} X, \quad X H_{\nu}+H_{\nu} X \geq \eta_{\nu} X \\
L_{\mu} \geq \varpi_{\mu} X, \quad L_{\nu} \geq \varpi_{\nu} X, \quad X>0
\end{array}\right.
$$

be feasible for $R_{\mu}=\left(\begin{array}{cc}0_{n-1} & 0 \\ 0 & r_{\mu}\end{array}\right), R_{\nu}=\left(\begin{array}{cc}0_{n-1} & 0 \\ 0 & r_{\nu}\end{array}\right)$ and some $\nu \in \mathbb{R}_{\geq}, \mu \in(0,1], \iota, \eta_{\mu}, \eta_{\nu}, r_{\mu}, r_{\nu}, \zeta, \varpi_{\mu}, \varpi_{\nu} \in \mathbb{R}_{>}$, 
$X \in \mathbb{R}^{n \times n}, y \in \mathbb{R}^{1 \times n}$ and the control law $u$ is chosen in the form

$$
u(V, x)=\left\{\begin{array}{lll}
V^{1-\mu} k D_{\mu}\left(V^{-1}\right) x & \text { for } & x^{T} P x \geq 1, \\
V^{1+\nu} k D_{\nu}\left(V^{-1}\right) x & \text { for } & x^{T} P x<1,
\end{array}\right.
$$

where $k=y X^{-1}, P=X^{-1}$ and $V$ is defined by

$$
V \in \mathbb{R}_{>}:\left\{\begin{array}{lll}
Q_{1}(V, x)=0 & \text { for } & x^{T} P x \geq 1, \\
Q_{2}(V, x)=0 & \text { for } & x^{T} P x<1 .
\end{array}\right.
$$

Then the origin of (2), (19) is globally asymptotically stable if

$$
\begin{gathered}
\tau<\min \left\{\frac{\sqrt{r_{\mu} \varpi_{\mu}}}{\eta_{\mu}^{-1} \sqrt{l_{1_{\mu}}}\left(\iota+2 \sqrt{p_{n n} \zeta}\right)+\sqrt{l_{2}}+\left|k_{n}\right| \sqrt{\zeta}},\right. \\
\left.\frac{\sqrt{r_{\nu} \varpi_{\nu}}}{\eta_{\nu}^{-1} \sqrt{l_{1_{\nu}}}\left(\iota+2 \sqrt{p_{n n} \zeta}\right)+\sqrt{l_{2}}+\left|k_{n}\right| \sqrt{\zeta}}\right\},
\end{gathered}
$$

where $l_{1_{\mu}}=\lambda_{\max }\left(X^{1 / 2}\left((1-\mu) I+H_{\mu}\right) k^{T} k((1-\mu) I+\right.$ $\left.\left.H_{\mu}\right) X^{1 / 2}\right), l_{1_{\nu}}=\lambda_{\max }\left(X^{1 / 2}\left((1+\nu) I+H_{\nu}\right) k^{T} k((1+\nu) I+\right.$ $\left.\left.H_{\nu}\right) X^{1 / 2}\right)$ and $l_{2}=\lambda_{\max }\left(X^{1 / 2} A^{T} k^{T} k A X^{1 / 2}\right)$. Moreover, for any $\tau>0$ trajectories of the closed-loop system are globally bounded.

Theorem 5 proves very important advantage of nonlinear control (19). Once designed it remains robust (in ISS sense) with respect to any delay.

Example 3 Consider the system (2) for $n=3$ in the disturbance-free case $(d(t, x(t))=0)$. Define the control $u$ in the form (19) with the parameters $\mu=0.5, \nu=0.2$. The matrix $P>0$ and the vector $k$ are obtained from the system (22) with $r_{\mu}=1.005, r_{\nu}=2.5001, \eta_{\mu}=\eta_{\nu}=1.5$, $\varpi_{\mu}=\varpi_{\nu}=1$ :

$$
\begin{gathered}
P=\left(\begin{array}{lll}
0.4011 & 0.8020 & 0.4007 \\
0.8020 & 2.0042 & 1.2018 \\
0.4007 & 1.2018 & 1.2013
\end{array}\right), \\
k=\left(\begin{array}{lll}
-1.0016 & -3.0037 & -3.0022
\end{array}\right) .
\end{gathered}
$$

Obtained value of maximal delay is $\tau_{0}=\min \{2.78 \times$ $\left.10^{-2}, 3.16 \times 10^{-2}\right\}=2.78 \times 10^{-2}$. According to Theorem 5 the system (2), (19) is globally asymptotically stable if $\tau<\tau_{0}=2.78 \times 10^{-2}$. Otherwise, similarly to Lemma 3 the system is converging to some compact set around the origin. The simulation results for $\tau=2.75 \times 10^{-2}$ are shown on Fig. 1 .

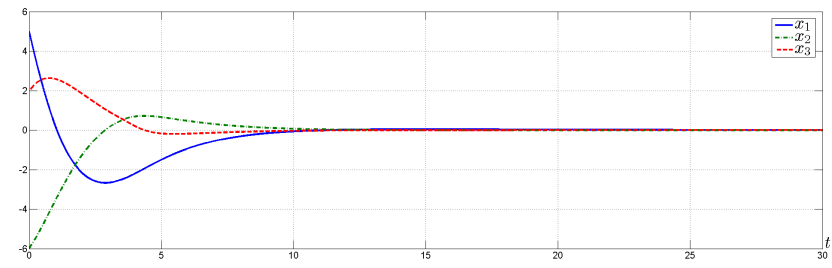

Fig. 1. The simulation results for the ILF control (19)
Theorem 6 (Disturbed case) Let for (2) the system of linear matrix inequalities

$$
\left\{\begin{array}{l}
\kappa_{\mu}\left(A X+X A^{T}+b y+y^{T} b^{T}\right)+L_{\mu_{1}}+R_{\mu_{1}} \leq 0 \\
\left(1-\kappa_{\mu}\right)\left(A X+X A^{T}+b y+y^{T} b^{T}\right)+L_{\mu_{2}}+R_{\mu_{2}} \leq 0 \\
\kappa_{\nu}\left(A X+X A^{T}+b y+y^{T} b^{T}\right)+L_{\nu_{1}}+R_{\nu_{1}} \leq 0 \\
\left(1-\kappa_{\nu}\right)\left(A X+X A^{T}+b y+y^{T} b^{T}\right)+L_{\nu_{2}}+R_{\nu_{2}} \leq 0 \\
\iota X \geq A X+X A^{T} \geq-\iota X, \quad\left(\begin{array}{c}
X \\
y \\
y
\end{array}\right) \geq 0 \\
X H_{\mu}+H_{\mu} X \geq \eta_{\mu} X, X H_{\nu}+H_{\nu} X \geq \eta_{\nu} X, X>0 \\
L_{\mu_{1}} \geq \varpi_{\mu} X, \quad L_{\mu_{2}} \leq \varpi_{\mu_{2}} X, \quad R_{\mu_{2}} \leq \varpi_{\mu_{3}} X \\
L_{\nu_{1}} \geq \varpi_{\nu} X, \quad L_{\nu_{2}} \leq \varpi_{\nu_{2}} X, \quad R_{\nu_{2}} \leq \varpi_{\nu_{3}} X
\end{array}\right.
$$

be feasible for $R_{\mu_{1}}=\left(\begin{array}{cc}0_{n-1} & 0 \\ 0 & r_{\mu}\end{array}\right), R_{\nu_{1}}=\left(\begin{array}{cc}0_{n-1} & 0 \\ 0 & r_{\nu}\end{array}\right)$ and some $\kappa_{\mu} \in(0,1), \kappa_{\nu} \in(0,1), \nu \in \mathbb{R}_{>}, \mu \in(0,1]$, $\iota, \zeta, \eta_{\mu}, \eta_{\nu}, r_{\mu}, r_{\nu}, \varpi_{\mu}, \varpi_{\nu} \in \mathbb{R}_{>}, R_{\mu_{2}}>0, R_{\nu_{2}}>0$, $X \in \mathbb{R}^{n \times n}, y \in \mathbb{R}^{1 \times n}$, the control law $u$ is chosen in the form (19) with $k=y X^{-1}, P=X^{-1}$ and the disturbance function $d(t, x)$ satisfies

$$
\begin{aligned}
& d^{T}(t, x) D_{\mu}\left(V^{-1}\right) R_{\mu_{2}}^{-1} D_{\mu}\left(V^{-1}\right) d(t, x) \leq \\
& \beta_{\mu} V^{-2 \mu} x^{T} D_{\mu}\left(V^{-1}\right) P L_{\mu_{2}} P D_{\mu}\left(V^{-1}\right) x \quad \text { if } \quad x^{T} P x \geq 1, \\
& d^{T}(t, x) D_{\nu}\left(V^{-1}\right) R_{\nu_{2}}^{-1} D_{\nu}\left(V^{-1}\right) d(t, x) \leq \\
& \quad \beta_{\nu} V^{2 \nu} x^{T}(t) D_{\nu}\left(V^{-1}\right) P L_{\nu_{2}} P D_{\nu}\left(V^{-1}\right) x \quad \text { if } \quad x^{T} P x<1,
\end{aligned}
$$

where $\beta_{\mu} \in(0,1), \beta_{\nu} \in(0,1)$. Then the origin of (2), (19) is globally asymptotically stable if

$$
\begin{gathered}
\tau< \\
\min \left\{\frac{\sqrt{r_{\mu} \varpi \mu}}{\eta_{\mu}^{-1} \sqrt{l_{1 \mu}}\left(\iota+2 \sqrt{p_{n n} \zeta}+2 \sqrt{\varpi \mu_{2} \varpi \mu_{3}}\right)+\sqrt{l_{2}}+\left|k_{n}\right| \sqrt{\zeta}+\sqrt{\zeta \varpi \mu_{2} \varpi \mu_{3}}},\right. \\
\\
\left.\quad \frac{\sqrt{r_{\nu} \varpi \nu}}{\eta_{\nu}^{-1} \sqrt{l_{1_{\nu}}}\left(\iota+2 \sqrt{p_{n n} \zeta}+2 \sqrt{\varpi \nu_{2} \varpi \nu_{3}}\right)+\sqrt{l_{2}}+\left|k_{n}\right| \sqrt{\zeta}+\sqrt{\zeta \varpi_{\nu_{2}} \varpi_{\nu_{3}}}}\right\},
\end{gathered}
$$

where $l_{1_{\mu}}=\lambda_{\max }\left(X^{1 / 2}\left((1-\mu) I+H_{\mu}\right) k^{T} k((1-\mu) I+\right.$ $\left.\left.H_{\mu}\right) X^{1 / 2}\right), l_{1_{\nu}}=\lambda_{\max }\left(X^{1 / 2}\left((1+\nu) I+H_{\nu}\right) k^{T} k((1+\nu) I+\right.$ $\left.\left.H_{\nu}\right) X^{1 / 2}\right)$ and $l_{2}=\lambda_{\max }\left(X^{1 / 2} A^{T} k^{T} k A X^{1 / 2}\right)$. Moreover, for any $\tau>0$ trajectories of the closed-loop system are globally bounded.

Example 4 Consider the system (2) for $n=3$ with disturbances $d_{1}(t, x(t))=0, d_{2}(t, x(t))=0, d_{3}(t, x(t))=$ $0.2 \sin \left(\left|x_{2}\right|^{0.5}\right)$. Define the control $u(t)$ in the form (19) with the parameters $\mu=1, \nu=0$. The matrix $P>0$ and the vector $k$ are obtained from the system (22) with $R_{\mu_{2}}=\left(\begin{array}{cc}0_{2} & 0 \\ 0 & 0.201\end{array}\right), R_{\nu_{2}}=\left(\begin{array}{cc}0_{2} & 0 \\ 0 & 0.445\end{array}\right), r_{\mu}=0.804$, $r_{\nu}=2.0551, \eta_{\mu}=1.8, \eta_{\nu}=1.5, \varpi_{\mu}=\varpi_{\nu}=1, \kappa_{\mu}=0.8$, $\kappa_{\nu}=0.822$ :

$$
\begin{gathered}
P=\left(\begin{array}{lll}
1.3676 & 2.2118 & 0.5187 \\
2.2118 & 4.8663 & 1.4809 \\
0.5187 & 1.4809 & 1.4804
\end{array}\right), \\
k=\left(\begin{array}{lll}
-1.9671 & -4.9881 & -3.3510
\end{array}\right) .
\end{gathered}
$$

Since $\sin \left(x_{2}\right) \leq\left|x_{2}\right|$ it can be easily shown that inequalities (23) hold. Obtained value of maximal delay is $\tau_{0}=$ $\min \left\{2.04 \times 10^{-2}, 2.65 \times 10^{-2}\right\}=2.04 \times 10^{-2}$. According to Theorem 6 the system (2), (19) is globally asymptotically stable if $\tau<\tau_{0}=2.04 \times 10^{-2}$. Otherwise, similarly to the result of Lemma 4 the system is converging to some compact set around the origin. The simulation results for constant delay $\tau=2 \times 10^{-2}$ are shown on Fig.2. 


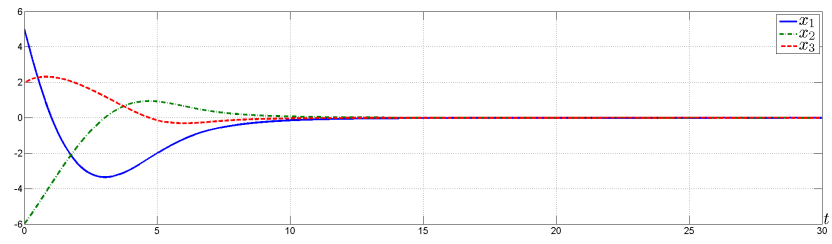

Fig. 2. The simulation results for the ILF control (19)

Presented results may be of interest for system protection against unforeseen increase of the delay. To appreciate the need for such protection, let us run a scenario of events that could happen in the operation of a system. Suppose that for the system in the form (2) some developed control law shows a good performance for all $(x, \tau) \in D_{w s} \times\left[0, \tau_{1}\right)$, where $D_{w s}$ is a domain containing the origin $\left(D_{w s} \subset R_{A}\right.$, where $R_{A}$ is a region of attraction). For example, it can be linearly controlled system developed for the delay-free case and investigated for the delayed control case (for instance, using results of [4]). Due to simplicity, such control laws are widely used in practice. Suppose the system is operating at steady state. If at some time $t_{0}$ a load increasing on the data transfer channel takes place and consequently delay value increases to $\tau \geq \tau_{1}$. Then, the trajectory of the system will be driven away the origin until the delay became again less than $\tau_{1}$ at some time instant $t_{1}$. Then the system will return to steady-state operation only if $x\left(t_{1}\right)$ belongs to the region of attraction of the origin $R_{A}$. Even if $R_{A}=\mathbb{R}^{n}$, behavior of the unstable system can lead to malfunctions and faults. Such a scenario is typical for the case of remote control, when the data transmission channel is fickle (for example, when control is via the Internet). To avoid such a situation, in addition to presented control laws another way to use obtained results for system protection is development of discontinuous event-triggered control law based on combination of the control (7) with another nonlinear control $u_{n l}$, which shows a good performance for the delay-free/small delay cases:

$$
u=\left\{\begin{array}{l}
V^{1-\mu} k D_{\mu}\left(V^{-1}\right) x \quad \text { for } \quad x \notin D_{w s}, \\
u_{n l} \text { for } x \in D_{w s} .
\end{array}\right.
$$

While the delay is larger than it is preferable for using the control $u_{n l}$, the control (25) allows to keep systems' trajectories in a compact set around the origin.

\section{CONCLUSIONS}

The paper presents ILF-based control algorithms for systems with the delay in control (and/or measurement) channel, i.e. teleoperated and networked systems. Obtained nonlinear control algorithms preserve asymptotic stability for small delays $0<\tau<\tau_{0}<\infty$ and global asymptotic stability with respect to a compact set containing the origin for any $\tau \geq \tau_{0}$. The results can be used for the cases of a constant and fastvarying delays. Efficiency of the proposed control algorithms is demonstrated on numerical examples. There are a lot of topics for future research. For example, relaxation of restrictions, extension of the results for MIMO systems, etc.

\section{REFERENCES}

[1] J.-P. Richard, Time-delay systems: an overview of some recent advances and open problems, Automatica, vol. 39(10), pp. 1667-1694, 2003.

[2] L. Bushnell, Editorial: Networks and control, IEEE Control System Magazine, vol. 21(1), pp. 22-99.

[3] C.-C. Hua, X.P. Liu, Editorial: Delay-Dependent Stability Criteria of Teleoperation Systems With Asymmetric Time-Varying Delays, IEEE Transactions on Robotics, vol. 26(5), pp. 925-932, 2010.

[4] E. Fridman, Tutorial on Lyapunov-based methods for time-delay systems, European Journal of Control, vol. 20(6), pp. 271-283, 2014

[5] T. Slama, A. Trevisani, D. Aubry, R. Oboe, F. Kratz, Experimental Analysis of an Internet-Based Bilateral Teleoperation System With Motion and Force Scaling Using a Model Predictive Controller, IEEE Transactions on Industrial Electronics, vol. 55(9), pp. 3290-3299, 2008

[6] S. Hirche, M. Buss, Human-Oriented Control for Haptic Teleoperation, Proceedings of the IEEE, vol. 100(3), pp. 623-647, 2012.

[7] E. Fridman, Introduction to Time-Delay Systems: Analysis and Control, Birkhuser, Basel, 2014.

[8] Yu. Mikheev, V. Sobolev, E. Fridman, Asymptotic analysis of digital control systems, Autom. Remote Control, vol. 49, pp. 1175-1180, 1988

[9] A. Isidori, Nonlinear Control Systems II, Springer-Verlag London, 293 p., 1999.

[10] A. Filippov, Differential equations with discontinuous right-hand sides, Dordrecht, Kluwer, 1988.

[11] E. Cruz-Zavala, J.A. Moreno, L. Fridman, Uniform Second-Order Sliding Mode Observer for mechanical systems, 11th International Workshop on Variable Structure Systems, 2010.

[12] M. Livne, A. Levant, Accuracy of disturbed homogeneous sliding modes, the 13th Scientific Workshop VSS13, 2014.

[13] A. Levant, Homogeneity of differential inclusions: Application to sliding modes, European Control Conference (ECC 2015), pp. 2458 2463,2015

[14] A. Polyakov, D. Efimov, W. Perruquetti, Robust Stabilization of MIMO Systems in Finite/Fixed Time, Int. J. Robust. Nonlinear Control, DOI:10.1002/rnc.3297, 2016.

[15] Y. Orlov, Finite Time Stability and Robust Control Synthesis of Uncertain Switched Systems, SIAM Journal of Control and Optimization, vol. 43(4), pp. 1253-1271, 2004

[16] V.I. Korobov, A general approach to synthesis problem, Doklady Academii Nauk SSSR, vol. 248, pp. 1051-1063, 1979.

[17] J. Adamy, A. Flemming, Soft variable-structure controls: a survey, Automatica, vol. 40, pp. 1821-1844, 2004.

[18] D. Efimov, W. Perruquetti, Homogeneity for time-delay systems, In Proc. IFAC WC 2011, Milan, 2011.

[19] A. Bacciotti, L. Rosier, Lyapunov Functions and Stability in Control Theory, Springer, 237 p., 2005.

[20] D. Efimov, A. Polyakov, W. Perruquetti, and J.-P. Richard, Weighted homogeneity for time-delay systems: Finite-time and independent of delay stability, IEEE Trans. Automatic Control, vol. 61(1): 210-215, 2016

[21] V.B. Kolmanovsky and V.R. Nosov, Stability of functional differential equations. CA: Academic, San Diego, 1986.

[22] K. Zimenko, D. Efimov, A. Polyakov, and W. Perruquetti, A note on delay robustness for homogeneous systems with negative degree, Automatica, vol. 79, pp. 178-184, 2017.

[23] A. Polyakov, D. Efimov, and W. Perruquetti, Finite-time and fixedtime stabilization: Implicit Lyapunov function approach, Automatica, vol. 51, pp. 332-340, 2015.

[24] S. Bhat, D. Bernstein, Finite-time stability of continuous autonomous systems, SIAM Journal of Control and Optimization, vol. 38(3), pp. 751-766, 2000

[25] S. Laghrouche, M. Harmouche, Y. Chitour, Stabilization of perturbed integrator chains using Lyapunov-Based Homogeneous Controllers, International Journal of Control, pp. 1-10, 2016. 